\title{
The Predictability of GARCH-Type Models on the Returns Volatility of Primary Indonesian Exported Agricultural Commodities
}

\author{
Saarce Elsye Hatane \\ Accounting Department, Petra Christian University \\ Email: elsyehat@peter.petra.ac.id
}

\begin{abstract}
Agricultural sector plays an important role in Indonesia's economy; especially for the plantation sub-sector contributing high revenues to Indonesia's exporting sectors. The primary agricultural commodities in Indonesian export discussed in this study would be Crude Palm Oil (CPO), Natural Rubber TSR20, Arabica Coffee, Robusta Coffee, Cocoa, White Pepper and Black Pepper. Meanwhile, the returns volatility nature of agricultural commodity is famous. The volatility refers to heteroscedasticity nature of the returns which can be modeled by GARCH-type models. The returns volatility can be describe by the residual of the mean equation and volatility of error variances in the previous periods. The aims of this study are to examine the predictability of GARCH-type models on the returns volatility of those seven agricultural commodities and to determine the best GARCH-type models for each commodity based on the traditional symmetric evaluation statistics. The results find that the predictability of ARCH, GARCH, GARCH-M, EGACRH and TGARCH, as type of GARCH models used in this study, are different for each commodity.
\end{abstract}

Keywords: ARCH, GARCH, GARCH-M, EGACRH, TGARCH, returns volatility, residuals, agricultural commodity.

\section{INTRODUCTION}

Low returns and high risks are two issues faced by agricultural commodity producers as mentioned by UK's Department for International Development (DFID) on its 2004 report. According to the DFID, those two problems occur due to the less rapid growth of agricultural commodities' prices compared to those of manufactured products, and the price volatility in agricultural commodities. Generally, commodities, especially agricultural commodities, are well known for their prices volatility patterns (Newbery, 1989). The DFID argues that developing countries which are highly dependable on agricultural commodities should try to decrease their dependency on those commodeties. One such developing country, which will be the focus of this article, is Indonesia. Agriculture is traditionally the main sector of Indonesia's economic activity. Until 1960, it represented 50\% of Indonesian's Gross Domestic Product (GDP), as shown in Figure 1. The decline of the percentage inthe GDP since 1966 was due to the effort to reduce Indonesian overdependence on farmers. In the period of OrdeBaru, which was headed by Soeharto (Indonesian second president), the economy development were not just focused on agriculture, but also on other sectors such as manufacturing, electricity, construction, services and finance sectors (Djamin, 1989). Figure 1 shows that in the percentage of agriculture roles on GDP decreased from about 50\% in 1960 to about $16 \%$ in 1996. The percentage increased to be $18 \%$ in 1998 and $19 \%$ in 1999.

Three decades of stable progress in Indonesian agricultural development were suddenly disturbed by financial and environmental shocks in 1997 (Daryanto, 1999). Daryanto also mentioned that those conditions caused food insecurity, but in opposite to food crops, the Asian crisis gave positive impacts on farm non-food crops (plantations) and forestry. As high export-oriented and low importoriented subsectors, they enjoyed the prizes from the Asian crisis due to the Indonesian Rupiah (IDR) depreciation. After the Asian financial crisis in 1998, the good performance of agricultural exports was one factor, from agricultural sector, that saved Indonesia from the crisis (Basri, 2002).

Discussion on the degree of commodity price volatility has become one remarkable topic, and 


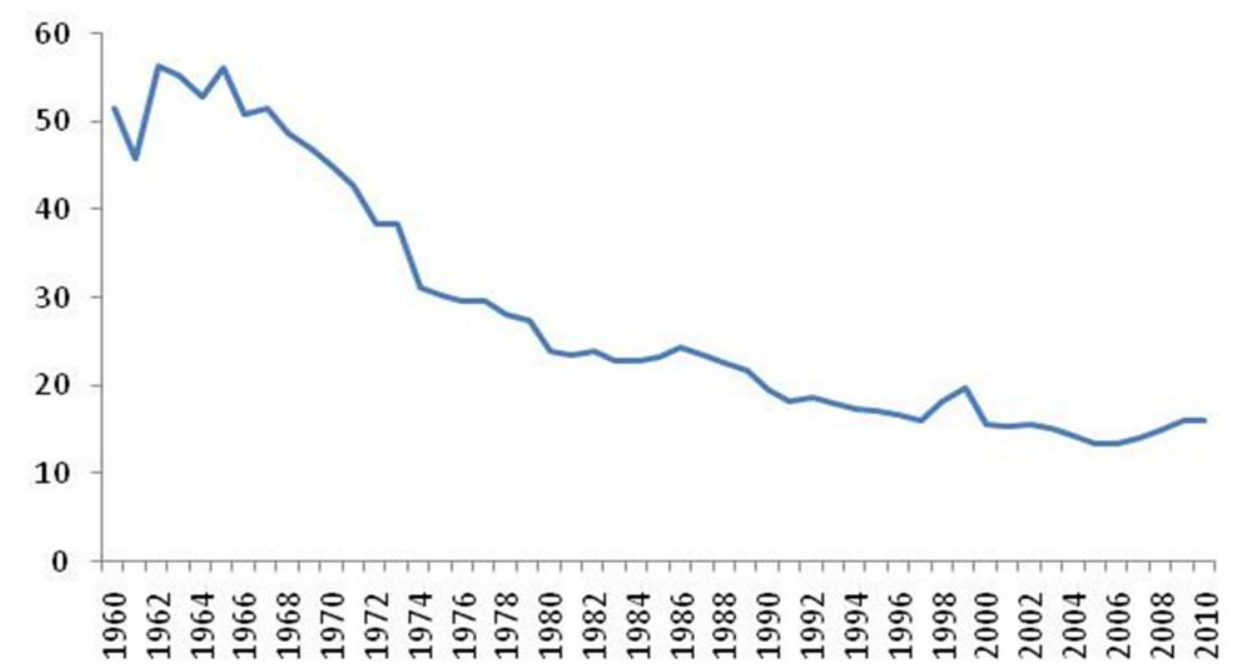

Source: Indicators Data of the World Bank. Data was modified by the author.

Figure 1. Indonesian Agriculture, Value Added - \% of GDP

attracted attention of researchers in economic and financial fields. For instance, Kroner et al. (1993); Sekhar (2003, 2004); O'Connor et al. (2009); and Alom et al. (2010), they reported that international prices of agricultural commodities are one of the most volatile prices in international market. Deaton (1999) argued that having a better understanding about commodity prices characteristics is extremely important for developing countries that depend on commodity exports or that import huge amounts of food. Many researchers have employed and extended the ARCH/GARCH methodology to examine various commodities price volatility issues. For example, Alom et al. (2010); Sumaryanto (2009); O'Connor et al. (2009); Zheng et al. (2008); Apergis and Rezitis (2003, 2011); Yang et al. (2001); and Beck (2001), theyapplied GARCHtype models to analyze the price volatility of agricultural products. Some empirical studies reported the existence of price volatility in futures prices and spot prices of some commodities. Mahesha (2011) reported that international spot prices of cardamom, ginger and pepper from India indicated long persistence and volatility clustering. Yang et al. (2001) also reported that some US commodities, i.e. corn, oat, soybeans, wheat and cotton, had price volatility feature, both for futures prices and spot prices.

\section{Measuring and Forcasting Volatility}

Volatility comes from the term of 'volatile'. This term refers to conditions that unstable prices tend to vary and are difficult to forecast. The key words in volatility are variability and uncertainty (Engle, 2003). Volatility is an important variable for port- folio management, option pricing and market regulations (Poon and Granger, 2003). The relationship between volatility and option price is positive. When volatility increases, then the option price will also increase. Thereby, information about price volatility is useful in estimating more precise and reasonable option price. Some empirical studies reveal that price volatility can be measured by standard deviation and coefficient of variation of asset price concerned.

According to Engle (1982), Bollerslev (1986) and Taylor (1986), the particular non-linear models that have been proven very useful in finance are the autoregressive conditional heteroscedasticity $(\mathrm{ARCH})$ and generalized autoregressive conditional heteroscedasticity (GARCH) models.

Thus far, the forecasting methods of time series data are autoregressive (AR), moving average (MA), or a combination of both (either ARMA or ARIMA). Results obtained from those forecasting methods will have high accuracy if the assumption of homoscedasticity in the error variances fulfilled. However, some problems arise when those forecasting methods are applied to commodity market which its price fluctuations tend to be bunches, like happened in stock exchange market or futures exchange market. The bunched features characterized the existence of large changes (e.g. large returns) are expected to follow large changes, and conversely, small changes to follow small changes (Diebold, 2004). These characteristics are known as heteroscedasticity. In time series data which have heteroscedasticity variances, the variances of error do not depend on their independent variable. Those variances are changing along with the time change. Those time series data 
have volatility character which is heteroscedasticity, because their error variances depend on the volatility of past errors. Data that have heteroscedasticity nature can be modeled by $\mathrm{ARCH}$ and GARCH models. ARCH/GARCH models utilize heteroscedasticity in the error variance appropriately in order to get the more efficient estimators. Good AR and MA models can be suitable for ARCH models, particularly in modeling the means' changes (Shephard, 1996).

\section{Previous Study}

In 2001, Beck analyzed the ARCH process for twenty commodities, storable and non-storable commodities, by using annual spot market data.. The results showed that prices volatility of each commodity was modeled by different type of ARCH/GARCH models. In summary, price volatility which was examined by ARCH/GARCH models mostly found in storable commodities. Sumaryanto (2009) analyzed retail price volatility of some Indonesian food commodities using ARCH/ GARCH models. From the overall estimateon results, it appeared that the most appropriate model for rice, red chili and shallot was ARCH (1); while for sugar and wheat flour was GARCH $(1,1)$. However, ARIMA was the fitted model for cooking oil and egg. Yang et al. (2001) examined the effect of agricultural liberalization policy, the Federal Agricultural Improvement and Reform (FAIR) Act of 1996, towards US agricultural commodity prices volatility using GARCH models. The commodities were corn, oat, soybeans, wheat and cotton. Total observations were 1695 active traded cash and futures prices from 1 January 1992 to 30 June 1998. Finally, the paper concluded that GARCH $(1,1)$ model had done adequate job in describing the data-generating process of cash and futures prices of each commodity. Mahesha (2011) investigated international price volatility of Indian of spices exports. This study applied GARCH $(1,1)$ model to estimate the time varying conditional variances. The result showed that there was a high volatility clustering in cardamom, ginger and pepper. Pinisakikool (2009) applied ARIMA-GARCH and ARIMA-TARCH with dummy variable to investigate whether futures traded in The Agricultural Futures Exchange of Thailand (AFET) could stabilize the spot price volatility or not. The results showed that spot price volatility model of the commodities studied were compatible with GARCH $(1,1)$ and TARCH $(2,2)$.

\section{RESEARCH METHODS}

The independent variables in this study are errors (residuals) from the mean equations (ARMA model) and volatility in the previous periods ( $t-1)$; while the dependent variable is the price returns volatility in current period $(t)$. The objects used in this study are CPO, Natural Rubber TSR20, Arabica Coffee, Robusta Coffee, Cocoa, White Pepper and Black Pepper. The specific purpose in this study is the predictability on GARCH-type models in describing the causal relationship between those variables. Since there are five type models of GARCH-type models used, which are ARCH; GARCH; GARCH-M; EGARCH; and TGARCH, and seven objects, this study does exploratory study to test whether those GARCHtype models can be used to predict the volatility of return prices of each commodity. All of the data used are weekly spot price series of those seven commodities from January 1, 2005 to June 30, 2011. The weekly spot price in this study is the closing price of immediate cash price on the last trading day of each week. Thus, total observations of each commodity are 338 weekly spot prices or equal to 337 observations of weekly spot price returns.

\section{Constructing ARMA Model}

Constructing ARMA model can be done only if the time series data is stationary. ARMA model is critical in generating a good GARCH forecasting model. There are four steps from Brooks (2008) used in this study to build the ARMA model. The identification process uses graphical procedures to determine the most appropriate specification. The graphics are plotting the data overtime and also the correlogram of autocorrelation function (ACF) and partial correlation function (PACF). ACF is used to the moving average (MA) model, while the PACF is used to predict the AR model. The estimation of ARMA models from the combination of AR and MA. The diagnostic process that testing the serial correlation problem and heteroscedasticity problem. The serial correlation problem should be solved first before testing the heteroscedasticity problem. From all of significant ARMA models, the best ARMA model was selected using Bayes Information Criterion (BIC) or known as Schwarz Information Criterion (SIC). The model with the lowest value of SIC should be chosen.

\section{The GARCH Building Process}

After determining the mean equation from ARMA model, the building of volatility equations in GARCH forms begins. The GARCH-type models employed in this study are:

ARCH (q) Model. It was proposed by Engle in 1982 to capture volatility persistence in inflation. 
The ARCH model does not utilize past standard deviations, but formulate conditional variance $\left(\sigma_{t}^{2}\right)$ of asset returns by maximum likelihood procedures. The conditional variance equation is:

$\sigma_{t}^{2}=a_{0}+a_{1} u_{t}^{2}-1+a_{2} u_{t}^{2}-2+\ldots+a_{q} u_{t}^{2}-q$

GARCH (p,q) Model.According to Bollerslev (1986) and Taylor (1986), the high-order ARCH(q) process is more proximate to model GARCH (p,q). The additional dependencies on the residual variance are permitted on $p$ lags of past $\sigma_{t}^{2}$ as shown below:

$\sigma_{t}^{2}=a_{0}+a_{1} u_{t-1}^{2}+\ldots+a_{q} u_{t-q}^{2}+\beta_{1} \sigma_{t-1}^{2}+\ldots+\beta_{p} \sigma_{t-p}^{2}$

GARCH-M (p, q) Model. It was introduced by Engle, Lilien and Robin in 1987, includes the conditional variance or standard deviation into the mean equation. The conditional variance equation is:

$\sigma_{t}^{2}=a_{0}+a_{1} u_{t-1}^{2}+\ldots+a_{q} u_{t-q}^{2}+\beta_{1} \sigma_{t-1}^{2}+\ldots+\beta_{p} \sigma_{t-p}^{2}$

EGARCH (p, q) Model. It was introduced by Nelson in 1991. The EGARCH (p,q) denotes conditional variance in logarithmic form. The equation is:

$$
\ln \left(\sigma_{t}^{2}\right)=\omega+\sum_{\mathrm{i}=1}^{\mathrm{p}} \beta_{i} \ln \left(\sigma_{t-i}^{2}\right)+\gamma \frac{u_{t-1}}{\sqrt{\sigma_{t-1}^{2}}}+\sum_{\mathrm{j}=1}^{\mathrm{p}} \alpha_{j}\left|\frac{u_{t-j}}{\sqrt{\sigma_{t-j}^{2}}}\right|
$$

TGARCH (p, q) Model.This model was introduced by Zakoïan in 1994. It was developed from Threshold Arch (TARCH or GJR) model by Glosten, Jaganathan and Runkle in 1993. The equation for conditional variance is:

$$
\begin{aligned}
& \sigma_{t}^{2}=\sigma_{0}+\sum_{\mathrm{i}=1}^{\mathrm{p}} \beta_{i} \sigma_{t-i}^{2}+\gamma u_{t-1} 1_{t-1}+\sum_{\mathrm{j}=1}^{\mathrm{q}} \alpha_{j} \mathrm{u}_{t-j}^{2} \\
& l_{t-k}=1 \text { if } u_{t-k}<0 ; l_{t-k}=0 \text { if } u_{t-k}>0
\end{aligned}
$$

Brooks (2008) explained three steps involved in estimating GARCH-type models. Determine the appropriate equations for the mean and the variance; determine the log-likelihood function (LLF) to maximize under a normality assumption for the disturbances; and computer program will maximize the function and generate parameter values that maximize the LLF and also will construct their standard errors.In order to have significant GARCH-type models, the probability value of each coefficient in those models has to be compared with critical values $(1 \%, 5 \%$ and $10 \%)$. If there is one insignificant coefficient in the estimated model, except the constant term, the null hypothesis will be failed to be rejected. It means that the model cannot be used to predict the volatility. In vice versa, if all of the coefficients in the model are significant, the null hypothesis will be rejected. It means that the model can be used to predict the volatility. However, this condition does not work in the constant term.

\section{The Evaluation Process}

The first step in evaluating the prediction power among GARCH-type models is measuring the "true or realized volatility." Brooks (2008) explained that true or ex post volatility is the actual historical volatility of a security's price. Ex post volatility measurement used in this study based on formula proposed by Day and Lewis (1992). The model is expressed as follow:

$\sigma_{t}^{2}=\left(\gamma_{t}-\gamma\right)^{2}$

The best predicting models among the GARCH-type models are selected by using three traditional symmetric evaluation statistics. Those are root mean square error (RMSE), mean absolute percent error (MAPE) and mean absolute error (MAE).

\section{RESULTS AND DISCUSSION}

The mean values, as shown in Table 1, were far from one. Those indicate that the data are stationary around zero. The standard deviation values, which are far from one, show the diversity of data which means that each commodity has high volatility in its price returns. The probability of Jarque-Bera in all of those commodities shows that those are not distributed normally. This study used $\mathrm{ADF}$ test statistic to perform the stationary test. The t-statistic value must be greater than the ADF test statistic values. Table 2 shows that all of those seven commodities have t-statistic values greater than all critical values. It means that the price return series of those commodities are stationary. The results of ARMA construction process for each commodity are shown in Table 3. Although Arabica, Robusta and Black Pepper are shown have no heteroscedasticity problem, the returns volatility of those commodities can still be predicted by $\mathrm{ARCH}$ family models (Francq \& Zakoian, 2010).

ARCH (q), GARCH (p, q), GARCH-M (p, q), $\operatorname{EGARCH}(p, q)$ and TGARCH $(p, q)$ were analyzed for each commodity. The $q$ expresses the lag of error or residual from the mean equation, while the $p$ expresses the lag of volatility. Each model is analyzed in four lags of residual and four lags of volatility. Table 4 to Table 10 shows the results of the variance equation for each commodity.

Table 4 shows that ARCH model; GARCH model; GARCH-M model; and TGARCH model can 
Table 1. Summary of Data Description

\begin{tabular}{|l|r|r|r|r|r|r|r|}
\hline Commodities & $\begin{array}{c}\text { Crude Palm } \\
\text { Oil (CPO) }\end{array}$ & \multicolumn{1}{c|}{$\begin{array}{l}\text { Natural } \\
\text { Rubber } \\
\text { TSR20 }\end{array}$} & \multicolumn{1}{c|}{$\begin{array}{l}\text { Arabica } \\
\text { Coffee }\end{array}$} & \multicolumn{1}{c|}{$\begin{array}{l}\text { Robusta } \\
\text { Coffee }\end{array}$} & Cocoa & \multicolumn{1}{c|}{$\begin{array}{l}\text { White } \\
\text { Pepper }\end{array}$} & Black Pepper \\
\hline Mean & 0.002518 & 0.003901 & 0.002427 & 0.003626 & 0.001571 & 0.003642 & 0.004304 \\
\hline Median & 0.004996 & 0.003517 & -0.000440 & 0.001121 & 0.001133 & 0.000838 & 0.000000 \\
\hline Maximum & 0.107354 & 0.202838 & 0.157131 & 0.241014 & 0.199190 & 0.405815 & 0.222508 \\
\hline Minimum & -0.175250 & -0.279927 & -0.139949 & -0.243196 & -0.203524 & -0.369170 & -0.084882 \\
\hline Std. Deviation & 0.040437 & 0.055393 & 0.039912 & 0.057288 & 0.047792 & 0.043246 & 0.033132 \\
\hline Skewness & -0.552174 & -0.856472 & 0.134207 & 0.380478 & 0.221388 & 0.581254 & 2.019041 \\
\hline Jarque-Bera & 57.22679 & 493.6266 & 41.13426 & 164.3157 & 84.01511 & 21166.58 & 1469.61 \\
\hline Probability & 0.000000 & 0.000000 & 0.000000 & 0.000000 & 0.000000 & 0.000000 & 0.000000 \\
\hline $\mathrm{N}$ & 337 & 337 & 337 & 337 & 337 & 337 & 337 \\
\hline
\end{tabular}

Table 2. Summary of Stationary Testing

\begin{tabular}{|l|c|c|c|c|c|}
\hline \multicolumn{1}{|c|}{ Commodities } & t-statistic & $\begin{array}{l}\text { Critical } \\
\text { Value at } \\
1 \% \text { level }\end{array}$ & $\begin{array}{l}\text { Critical } \\
\text { Value at } \\
5 \% \text { level }\end{array}$ & $\begin{array}{l}\text { Critical } \\
\text { Value at } \\
10 \% \text { level }\end{array}$ & Result \\
\hline Crude Palm Oil (CPO) & -17.9566 & -3.44962 & -2.86993 & -2.571307 & Stationary \\
\hline Natural Rubber TSR20 & -17.2618 & -3.44962 & -2.86993 & -2.571307 & Stationary \\
\hline Arabica Coffee & -18.5862 & -3.44962 & -2.86993 & -2.571307 & Stationary \\
\hline Robusta Coffee & -17.7254 & -3.44962 & -2.86993 & -2.571307 & Stationary \\
\hline Cocoa & -18.2729 & -3.44962 & -2.86993 & -2.571307 & Stationary \\
\hline White Pepper & -21.1486 & -3.44962 & -2.86993 & -2.571307 & Stationary \\
\hline Black Pepper & -14.8921 & -3.44962 & -2.86993 & -2.571307 & Stationary \\
\hline
\end{tabular}

Table 3. Summary of ARMA Models

\begin{tabular}{|c|c|c|c|c|c|c|c|}
\hline \multirow[b]{2}{*}{$\begin{array}{l}\text { ARMA Model of } \\
\text { Each Commodity }\end{array}$} & Crude Palm Oil & $\begin{array}{c}\text { Natural Rubber } \\
\text { TSR20 }\end{array}$ & $\begin{array}{l}\text { Arabica } \\
\text { Coffee }\end{array}$ & $\begin{array}{l}\text { Robusta } \\
\text { Coffee }\end{array}$ & Cocoa & White Pepper & $\begin{array}{l}\text { Black } \\
\text { Pepper }\end{array}$ \\
\hline & $\mathrm{AR}(32) \mathrm{MA}(32)$ & $\begin{array}{l}\mathrm{AR}(4) \operatorname{AR}(25) \\
\mathrm{MA}(25)\end{array}$ & $\begin{array}{l}\operatorname{AR}(28) \\
\operatorname{MA}(28)\end{array}$ & $\begin{array}{c}\operatorname{AR}(10) \\
\operatorname{MA}(10) \\
\operatorname{AR}(4)\end{array}$ & $\mathrm{AR}(21) \mathrm{MA}(21)$ & $\begin{array}{c}\mathrm{AR}(1) \mathrm{MA}(1) \mathrm{AR}(2) \\
\mathrm{MA}(2)\end{array}$ & $\begin{array}{c}\mathrm{AR}(1) \\
\mathrm{MA}(31)\end{array}$ \\
\hline $\begin{array}{l}\text { Serial Correlation } \\
\text { LM Test p-value } \\
\text { (Prob F) }\end{array}$ & 0.6502 & 0.6233 & 0.4464 & 0.8041 & 0.5804 & 0.1725 & 0.1912 \\
\hline $\begin{array}{l}\text { Serial Correlation } \\
\text { LM Test p-value } \\
\text { (Prob Chi-square) }\end{array}$ & 0.7314 & 0.7515 & 0.176 & 0.9991 & 0.6980 & 0.4160 & 0.5579 \\
\hline $\begin{array}{l}\text { Serial Correlation } \\
\text { Problem }\end{array}$ & No & No & No & No & No & No & No \\
\hline $\begin{array}{l}\text { ARCH LM Test p- } \\
\text { value (Prob F) }\end{array}$ & 0.0004 & 0.0000 & 0.4464 & 0.7587 & 0.0027 & 0.0000 & 0.7064 \\
\hline $\begin{array}{l}\text { ARCH LM Test p- } \\
\text { value (Prob Chi- } \\
\text { square) }\end{array}$ & 0.0005 & 0.0000 & 0.4415 & 0.7554 & 0.0029 & 0.0000 & 0.7029 \\
\hline $\begin{array}{l}\text { Heteroscedasticity } \\
\text { Problem }\end{array}$ & Yes & Yes & No & No & Yes & Yes & No \\
\hline Treatment & $\begin{array}{c}\text { White's } \\
\text { Heteroscedasticity }\end{array}$ & $\begin{array}{c}\text { White's } \\
\text { Heteroscedasticity }\end{array}$ & No & No & $\begin{array}{c}\text { White's } \\
\text { Heteroscedasticity }\end{array}$ & $\begin{array}{c}\text { White's } \\
\text { Heteroskedasticity }\end{array}$ & No \\
\hline SIC Value & -3.639561 & -3.047697 & -3.758862 & -2.902458 & -3.251513 & -3.47099 & -3.98940 \\
\hline
\end{tabular}


Table 4. The Results of GARCH Construction Process for CPO

\begin{tabular}{|c|c|c|c|c|c|c|c|c|c|c|}
\hline $\begin{array}{l}\text { GARCH-type } \\
\text { Models }\end{array}$ & $\alpha 0$ or $\omega$ & $\alpha 1$ & $\alpha 2$ & $\alpha 3$ & $\alpha 4$ & $\gamma$ & $\beta 1$ & $\beta 2$ & $\beta 3$ & $\beta 4$ \\
\hline $\mathrm{ARCH}$ (1) & $\begin{array}{c}0.001100^{*} \\
(0.0000) \\
\end{array}$ & $\begin{array}{c}0.271829^{* *} \\
(0.0120)\end{array}$ & & & & & & & & \\
\hline $\operatorname{GARCH}(1,1)$ & $\begin{array}{l}0.0000735 \\
(0.1000)\end{array}$ & $\begin{array}{c}0.103866^{\star *} \\
(0.0236)\end{array}$ & & & & & $\begin{array}{c}0.849031^{*} \\
(0.0000)\end{array}$ & & & \\
\hline $\operatorname{GARCH}(3,3)$ & $\begin{array}{c}0.000163^{\star \star} \\
(0.0449)\end{array}$ & $\begin{array}{c}0.131126^{\star *} \\
(0.0228)\end{array}$ & $\begin{array}{c}-0.094033^{\star *} \\
(0.0278)\end{array}$ & $\begin{array}{c}0.172872^{*} \\
(0.0019)\end{array}$ & & & $\begin{array}{c}0.709906^{*} \\
(0.0000)\end{array}$ & $\begin{array}{c}-0.659064^{*} \\
(0.0000)\end{array}$ & $\begin{array}{c}0.631783^{*} \\
(0.0000)\end{array}$ & \\
\hline $\operatorname{GARCH}-\mathrm{M}(1,1)$ & $\begin{array}{c}0.0000707^{* * *} \\
(0.0917)\end{array}$ & $\begin{array}{c}0.102932^{\star \star} \\
(0.0226) \\
\end{array}$ & & & & & $\begin{array}{c}0.851942^{*} \\
(0.0000) \\
\end{array}$ & & & \\
\hline GARCH-M $(3,3)$ & $\begin{array}{c}0.000158^{\star *} \\
(0.0412)\end{array}$ & $\begin{array}{c}0.134362^{\star \star} \\
(0.0215)\end{array}$ & $\begin{array}{c}-0.097256^{* *} \\
(0.0309)\end{array}$ & $\begin{array}{l}0.175471^{*} \\
(0.0021)\end{array}$ & & & $\begin{array}{l}0.69596^{*} \\
(0.0000) \\
\end{array}$ & $\begin{array}{c}-0.635278^{*} \\
(0.0000) \\
\end{array}$ & $\begin{array}{c}0.622947^{*} \\
(0.0000)\end{array}$ & \\
\hline TGARCH $(4,2)$ & $\begin{array}{c}0.000169^{*} \\
(0.0000)\end{array}$ & $\begin{array}{c}0.182262^{*} \\
(0.0001)\end{array}$ & $\begin{array}{c}0.077179^{* * *} \\
(0.0565)\end{array}$ & & & $\begin{array}{c}-0.058606^{\star * *} \\
(0.0582)\end{array}$ & $\begin{array}{c}-0.303633^{\text {** }} \\
(0.0261)\end{array}$ & $\begin{array}{c}0.951457^{*} \\
(0.0000)\end{array}$ & $\begin{array}{c}0.507213^{*} \\
(0.0000)\end{array}$ & $\begin{array}{r}-0.484314^{*} \\
(0.0002)\end{array}$ \\
\hline
\end{tabular}

Note: $*$ significant at $1 \% ;{ }^{* *}=$ significant at $5 \% ; * * *=$ significant at $10 \%$; value in the parenthesis is the p-value

Table 5. The Results of GARCH Construction Process for Natural Rubber TSR20

\begin{tabular}{|c|c|c|c|c|c|c|c|c|c|c|}
\hline $\begin{array}{c}\text { GARCH-type } \\
\text { Models }\end{array}$ & $\alpha 0$ or $\omega$ & $\alpha 1$ & $\alpha 2$ & $\alpha 3$ & $\alpha 4$ & $\gamma$ & $\beta 1$ & $\beta 2$ & $\beta 3$ & $\beta 4$ \\
\hline $\mathrm{ARCH}$ (1) & $\begin{array}{c}0.001590^{*} \\
(0.0000)\end{array}$ & $\begin{array}{c}0.459179^{\star *} \\
(0.0228)\end{array}$ & & & & & & & & \\
\hline $\mathrm{ARCH}(2)$ & $\begin{array}{c}0.001502^{*} \\
(0.0000)\end{array}$ & $\begin{array}{c}0.210533^{\star \star} \\
(0.0317)\end{array}$ & $\begin{array}{c}0.224205^{\star \star \star} \\
(0.0506)\end{array}$ & & & & & & & \\
\hline $\operatorname{GARCH}(1,1)$ & $\begin{array}{c}0.000379^{* *} \\
(0.0244)\end{array}$ & $\begin{array}{c}0.213271^{\star \star} \\
(0.0121) \\
\end{array}$ & & & & & $\begin{array}{c}0.652320^{*} \\
(0.0000)\end{array}$ & & & \\
\hline $\operatorname{GARCH}(2,1)$ & $\begin{array}{c}0.000157^{*} \\
(0.0005)\end{array}$ & $\begin{array}{c}0.092752^{* * *} \\
(0.0611) \\
\end{array}$ & & & & & $\begin{array}{c}1.438648^{*} \\
(0.0000)\end{array}$ & $\begin{array}{c}-0.584518^{*} \\
(0.0000) \\
\end{array}$ & & \\
\hline GARCH-M $(1,1)$ & $\begin{array}{c}0.000444^{*} \\
(0.0091)\end{array}$ & $\begin{array}{c}0.255309^{\star \star} \\
(0.0268)\end{array}$ & & & & & $\begin{array}{c}0.592863^{*} \\
(0.0000)\end{array}$ & & & \\
\hline GARCH-M $(2,1)$ & $\begin{array}{c}0.000175^{*} \\
(0.0010)\end{array}$ & $\begin{array}{c}0.110724^{\star \star \star} \\
(0.0658) \\
\end{array}$ & & & & & $\begin{array}{c}1.355094^{*} \\
(0.0000)\end{array}$ & $\begin{array}{c}-0.523508^{*} \\
(0.0002)\end{array}$ & & \\
\hline $\operatorname{EGARCH}(1,1)$ & $\begin{array}{c}-2.055568^{*} \\
(0.0047)\end{array}$ & $\begin{array}{c}0.416561^{*} \\
(0.0012) \\
\end{array}$ & & & & $\begin{array}{c}-0.186586^{* * *} \\
(0.0853) \\
\end{array}$ & $\begin{array}{c}0.710663^{*} \\
(0.0000)\end{array}$ & & & \\
\hline $\operatorname{EGARCH}(3,1)$ & $\begin{array}{c}-1.139105^{\star} \\
(0.0032)\end{array}$ & $\begin{array}{c}0.261171^{*} \\
(0.0000)\end{array}$ & & & & $\begin{array}{c}-0.120674^{\star} \\
(0.0052)\end{array}$ & $\begin{array}{c}1.623358^{*} \\
(0.0000)\end{array}$ & $\begin{array}{c}-1.601765^{\star} \\
(0.0000)\end{array}$ & $\begin{array}{c}0.822213^{\star} \\
(0.0000)\end{array}$ & \\
\hline
\end{tabular}

Note: ${ }^{*}=$ significant at $1 \% ; * *=$ significant at $5 \% ; * * *=$ significant at $10 \%$; value in the parenthesis is the $p$-value

Table 6. The Results of GARCH Construction Process for Arabica Coffee

\begin{tabular}{|c|c|c|c|c|c|c|c|c|c|c|}
\hline $\begin{array}{c}\text { GARCH-type } \\
\text { Models }\end{array}$ & $\alpha 0$ or $\omega$ & $\alpha 1$ & $\alpha 2$ & $\alpha 3$ & $\alpha 4$ & $\gamma$ & $\beta 1$ & $\beta 2$ & $\beta 3$ & $\beta 4$ \\
\hline $\operatorname{GARCH}(3,1)$ & $\begin{array}{c}0.000285^{\star \star *} \\
(0.0925)\end{array}$ & $\begin{array}{c}-0.0352918^{*} \\
(0.0000)\end{array}$ & & & & & $\begin{array}{c}1.638918^{*} \\
(0.0000)\end{array}$ & $\begin{array}{c}-1.568965^{\star} \\
(0.0000)\end{array}$ & $\begin{array}{c}0.752319^{*} \\
(0.0000)\end{array}$ & \\
\hline GARCH-M $(2,1)$ & $\begin{array}{c}0.001144^{*} \\
(0.0000)\end{array}$ & $\begin{array}{c}-0.021503^{\star \star} \\
(0.0443) \\
\end{array}$ & & & & & $\begin{array}{c}1.149463^{*} \\
(0.0000)\end{array}$ & $\begin{array}{c}-0.992513^{*} \\
(0.0000)\end{array}$ & & \\
\hline GARCH-M $(3,1)$ & $\begin{array}{c}0.000274 \\
(0.2112) \\
\end{array}$ & $\begin{array}{c}-0.030289^{*} \\
(0.0007)\end{array}$ & & & & & $\begin{array}{c}1.639019^{*} \\
(0.0000)\end{array}$ & $\begin{array}{c}-1.583797^{*} \\
(0.0000) \\
\end{array}$ & $\begin{array}{r}0.766305^{*} \\
(0.0000) \\
\end{array}$ & \\
\hline GARCH-M $(4,1)$ & $\begin{array}{c}0.000737^{\star \star *} \\
(0.0807)\end{array}$ & $\begin{array}{c}-0.026853^{*} \\
(0.0004)\end{array}$ & & & & & $\begin{array}{c}1.827113^{*} \\
(0.0000)\end{array}$ & $\begin{array}{c}-2.241016^{*} \\
(0.0000)\end{array}$ & $\begin{array}{c}1.372745^{\star} \\
(0.0023) \\
\end{array}$ & $\begin{array}{c}-0.496452^{* \star *} \\
(0.0913)\end{array}$ \\
\hline $\operatorname{EGARCH}(3,1)$ & $\begin{array}{c}-2.647366 \\
(0.2540)\end{array}$ & $\begin{array}{c}0.090598^{* \star \star} \\
(0.0686)\end{array}$ & & & & $\begin{array}{c}0.103701^{*} \\
(0.0066)\end{array}$ & $\begin{array}{c}0.857005^{*} \\
(0.0000)\end{array}$ & $\begin{array}{c}-1.009322^{*} \\
(0.0000)\end{array}$ & $\begin{array}{c}0.768157^{*} \\
(0.0000)\end{array}$ & \\
\hline $\operatorname{EGARCH}(4,1)$ & $\begin{array}{c}-16.677680^{*} \\
(0.0000)\end{array}$ & $\begin{array}{c}0.036383^{\star \star \star} \\
(0.0610) \\
\end{array}$ & & & & $\begin{array}{c}0.067865^{*} \\
(0.0001) \\
\end{array}$ & $\begin{array}{c}0.578589^{*} \\
(0.0000)\end{array}$ & $\begin{array}{c}-1.790400^{*} \\
(0.0000)\end{array}$ & $\begin{array}{c}0.544335^{\star} \\
(0.0000)\end{array}$ & $\begin{array}{c}-0.794044^{*} \\
(0.0000)\end{array}$ \\
\hline $\operatorname{TGARCH}(2,1)$ & $\begin{array}{c}0.000511^{*} \\
(0.0000)\end{array}$ & $\begin{array}{c}-0.038086^{*} \\
(0.0009)\end{array}$ & & & & $\begin{array}{c}-0.032398^{* *} \\
(0.0199)\end{array}$ & $\begin{array}{c}1.227330^{*} \\
(0.0000)\end{array}$ & $\begin{array}{c}-0.540441^{*} \\
(0.0020)\end{array}$ & & \\
\hline
\end{tabular}

Note: $*$ significant at $1 \% ; * *=$ significant at $5 \% ; * * *=$ significant at $10 \%$; value in the parenthesis is the $p$-value 
Table 7. The Results of GARCH Construction Process for Robusta Coffee

\begin{tabular}{|c|c|c|c|c|c|c|c|c|c|c|}
\hline $\begin{array}{l}\text { GARCH-type } \\
\text { Models }\end{array}$ & $\alpha 0$ or $\omega$ & $\alpha 1$ & $\alpha 2$ & $\alpha 3$ & $\alpha 4$ & $\gamma$ & $\beta 1$ & $\beta 2$ & $\beta 3$ & $\beta 4$ \\
\hline $\operatorname{GARCH}(1,1)$ & $\begin{array}{c}0.000248^{\star * \star} \\
(0.0826)\end{array}$ & $\begin{array}{c}0.034242^{\star * \star} \\
(0.0582)\end{array}$ & & & & & $\begin{array}{c}0.879238^{*} \\
(0.0000)\end{array}$ & & & \\
\hline $\operatorname{GARCH}(2,1)$ & $\begin{array}{c}0.0000846^{*} \\
(0.0000)\end{array}$ & $\begin{array}{c}0.011435^{*} \\
(0.0000)\end{array}$ & & & & & $\begin{array}{c}1.893306^{*} \\
(0.0000)\end{array}$ & $\begin{array}{c}-0.931270^{*} \\
(0.0000)\end{array}$ & & \\
\hline $\operatorname{GARCH}(3,1)$ & $\begin{array}{c}0.000437^{\star} \\
(0.0000)\end{array}$ & $\begin{array}{c}0.154229^{*} \\
(0.0000)\end{array}$ & & & & & $\begin{array}{c}0.086931^{*} \\
(0.0000)\end{array}$ & \begin{tabular}{|c}
$-0.180996^{*}$ \\
$(0.0000)$ \\
\end{tabular} & \begin{tabular}{|c|}
$0.810927^{\star}$ \\
$(0.0000)$ \\
\end{tabular} & \\
\hline $\operatorname{GARCH}(4,1)$ & $\begin{array}{c}0.000171^{*} \\
(0.0000)\end{array}$ & $\begin{array}{c}0.033694^{*} \\
(0.0000)\end{array}$ & & & & & $\begin{array}{l}1.03458^{*} \\
(0.0000)\end{array}$ & $\begin{array}{c}-0.289099^{*} \\
(0.0000)\end{array}$ & $\begin{array}{r}1.044142^{*} \\
(0.0000)\end{array}$ & $\begin{array}{c}-0.878824^{*} \\
(0.0000)\end{array}$ \\
\hline GARCH $(3,2)$ & $\begin{array}{c}0.000686^{* *} \\
(0.0140)\end{array}$ & $\begin{array}{l}0.05087^{\star} \\
(0.0017)\end{array}$ & $\begin{array}{c}0.108399^{*} \\
(0.0000)\end{array}$ & & & & $\begin{array}{c}-0.13602^{*} \\
(0.0000) \\
\end{array}$ & \begin{tabular}{|c}
$-0.091606^{\star \star}$ \\
$(0.0281)$ \\
\end{tabular} & \begin{tabular}{|c|}
$0.821833^{*}$ \\
$(0.0000)$ \\
\end{tabular} & \\
\hline $\operatorname{GARCH}(4,2)$ & $\begin{array}{c}0.000173^{*} \\
(0.0000)\end{array}$ & $\begin{array}{c}0.077102^{*} \\
(0.0000) \\
\end{array}$ & $\begin{array}{c}0.054868^{*} \\
(0.0000)\end{array}$ & & & & $\begin{array}{c}0.371196^{*} \\
(0.0000)\end{array}$ & $\begin{array}{c}0.312544^{*} \\
(0.0000) \\
\end{array}$ & $\begin{array}{r}0.799405^{*} \\
(0.0000) \\
\end{array}$ & $\begin{array}{c}-0.663282^{*} \\
(0.0000)\end{array}$ \\
\hline GARCH-M $(1,1)$ & $\begin{array}{c}0.000245^{\star \star \star} \\
(0.0827)\end{array}$ & $\begin{array}{c}0.033193^{\star \star \star} \\
(0.0749)\end{array}$ & & & & & $\begin{array}{l}0.88106^{\star} \\
(0.0000)\end{array}$ & & & \\
\hline GARCH-M $(2,1)$ & $\begin{array}{c}0.0000829^{\star} \\
(0.0000)\end{array}$ & $\begin{array}{c}0.011920^{\star} \\
(0.0000)\end{array}$ & & & & & $\begin{array}{c}1.890689^{*} \\
(0.0000)\end{array}$ & \begin{tabular}{|c}
$-0.928651^{\star}$ \\
$(0.0000)$ \\
\end{tabular} & & \\
\hline GARCH-M $(3,1)$ & $\begin{array}{c}0.000529^{\star} \\
(0.0070)\end{array}$ & $\begin{array}{c}0.138685^{\star} \\
(0.0000)\end{array}$ & & & & & $\begin{array}{c}0.062905^{\star} \\
(0.0077)\end{array}$ & \begin{tabular}{|c|}
$-0.180909^{*}$ \\
$(0.0000)$
\end{tabular} & $\begin{array}{c}0.808576^{\star} \\
(0.0000) \\
\end{array}$ & \\
\hline GARCH-M $(4,1)$ & $\begin{array}{c}0.000104^{*} \\
(0.0000) \\
\end{array}$ & $\begin{array}{c}0.015128^{*} \\
(0.0000) \\
\end{array}$ & & & & & $\begin{array}{c}1.753363^{*} \\
(0.0000) \\
\end{array}$ & \begin{tabular}{|c|}
$-0.809277^{*}$ \\
$(0.0000)$ \\
\end{tabular} & \begin{tabular}{|c|}
$0.138283^{*}$ \\
$(0.0000)$ \\
\end{tabular} & $\begin{array}{c}-0.130318^{*} \\
(0.0000)\end{array}$ \\
\hline GARCH-M $(3,2)$ & $\begin{array}{c}0.000702^{* *} \\
(0.0154) \\
\end{array}$ & $\begin{array}{c}0.045508^{* *} \\
(0.0122) \\
\end{array}$ & $\begin{array}{c}0.115131^{*} \\
(0.0000)\end{array}$ & & & & $\begin{array}{c}-0.139939^{*} \\
(0.0000)\end{array}$ & $\begin{array}{c}0.080945^{* * *} \\
(0.0674) \\
\end{array}$ & $\begin{array}{c}0.818277^{*} \\
(0.0000)\end{array}$ & \\
\hline GARCH-M $(3,3)$ & $\begin{array}{c}0.000396^{\star} \\
0.0099\end{array}$ & $\begin{array}{c}0.104979^{*} \\
(0.0000)\end{array}$ & $\begin{array}{c}0.123503^{\star} \\
(0.0000)\end{array}$ & $\begin{array}{c}0.029245^{\star \star \star} \\
(0.0738) \\
\end{array}$ & & & $\begin{array}{c}-0.327306^{*} \\
(0.0000) \\
\end{array}$ & $\begin{array}{c}0.077822^{*} \\
0.0030 \\
\end{array}$ & \begin{tabular}{|c|}
$0.870776^{\star}$ \\
$(0.0000)$ \\
\end{tabular} & \\
\hline $\operatorname{EGARCH}(4,1)$ & $\begin{array}{c}-0.252494^{*} \\
(0.0000)\end{array}$ & $\begin{array}{c}-0.042667^{\star} \\
(0.0700)\end{array}$ & & & & $\begin{array}{c}0.072445^{\star} \\
(0.0433)\end{array}$ & $\begin{array}{c}0.420891^{*} \\
(0.0000))\end{array}$ & $\begin{array}{c}-0.179384^{*} \\
(0.0000)\end{array}$ & $\begin{array}{c}0.590311^{\star} \\
(0.0000)\end{array}$ & $\begin{array}{c}0.121910^{*} \\
(0.0000)\end{array}$ \\
\hline $\operatorname{EGARCH}(3,2)$ & $\begin{array}{c}-0.136467^{*} \\
(0.0000)\end{array}$ & $\begin{array}{c}-0.262761^{*} \\
(0.0000)\end{array}$ & $\begin{array}{c}0.018827^{*} \\
(0.0000)\end{array}$ & & & $\begin{array}{c}0.11701^{*} \\
(0.0007)\end{array}$ & $\begin{array}{c}-0.263653^{*} \\
(0.0000)\end{array}$ & $\begin{array}{c}0.520553^{*} \\
(0.0000)\end{array}$ & \begin{tabular}{|c|}
$0.693092^{\star}$ \\
$(0.0000)$ \\
\end{tabular} & \\
\hline $\operatorname{EGARCH}(1,3)$ & $\begin{array}{c}-0.029515^{*} \\
(0.0000)\end{array}$ & $\begin{array}{c}-0.039061^{*} \\
(0.0000)\end{array}$ & $\begin{array}{c}0.231862^{*} \\
(0.0000)\end{array}$ & $\begin{array}{c}-0.263604^{*} \\
(0.0000)\end{array}$ & & $\begin{array}{c}0.038077^{\star \star \star} \\
(0.0860)\end{array}$ & $\begin{array}{c}0.987449^{*} \\
(0.0000)\end{array}$ & & & \\
\hline $\operatorname{TGARCH}(1,1)$ & $\begin{array}{c}0.000512^{*} \\
(0.0017)\end{array}$ & $\begin{array}{c}0.070194^{* * *} \\
(0.0937)\end{array}$ & & & & $\begin{array}{c}-0.126044^{*} \\
(0.0066)\end{array}$ & $\begin{array}{c}0.807042^{*} \\
(0.0000)\end{array}$ & & & \\
\hline
\end{tabular}

Note: $*$ significant at $1 \% ;{ }^{* *}=$ significant at $5 \% ; * * *=$ significant at $10 \%$; value in the parenthesis is the p-value

Table 8. The Results of GARCH Construction Process for Cocoa

\begin{tabular}{|c|c|c|c|c|c|c|c|c|c|c|}
\hline $\begin{array}{c}\text { GARCH-type } \\
\text { Models }\end{array}$ & $\alpha 0$ or $\omega$ & $\alpha 1$ & $\alpha 2$ & $\alpha 3$ & $\alpha 4$ & $\gamma$ & $\beta 1$ & $\beta 2$ & $\beta 3$ & $\beta 4$ \\
\hline $\operatorname{GARCH}(3,2)$ & $\begin{array}{c}-0.00000137^{*} \\
(0.0000)\end{array}$ & $\begin{array}{c}0.103267^{*} \\
(0.0000)\end{array}$ & $\begin{array}{c}-0.107400^{*} \\
(0.0000) \\
\end{array}$ & & & & $\begin{array}{c}0.978809^{*} \\
(0.0000)\end{array}$ & $\begin{array}{c}0.910119^{*} \\
(0.0000) \\
\end{array}$ & \begin{tabular}{|c|}
$-0.884618^{*}$ \\
$(0.0000)$ \\
\end{tabular} & \\
\hline GARCH-M $(3,2)$ & $\begin{array}{c}0.00000127^{*} \\
(0.0000)\end{array}$ & $\begin{array}{c}0.099086^{*} \\
(0.0097)\end{array}$ & $\begin{array}{c}-0.099106^{* \star} \\
(0.0111)\end{array}$ & & & & $\begin{array}{c}0.973997^{*} \\
(0.0000)\end{array}$ & $\begin{array}{r}0.908512^{*} \\
(0.0000)\end{array}$ & $\begin{array}{c}-0.883058^{*} \\
(0.0000)\end{array}$ & \\
\hline $\operatorname{EGARCH}(3,2)$ & $\begin{array}{c}-1.398572^{\star \star \star} \\
(0.0619)\end{array}$ & $\begin{array}{c}0.284362^{*} \\
(0.0000)\end{array}$ & $\begin{array}{c}0.268115^{*} \\
(0.0000)\end{array}$ & & & $\begin{array}{c}0.064278^{*} \\
(0.0006)\end{array}$ & $\begin{array}{c}-0.808895^{\star} \\
(0.0000)\end{array}$ & $\begin{array}{r}0.718652^{*} \\
(0.0000) \\
\end{array}$ & $\begin{array}{c}0.933300^{*} \\
(0.0000)\end{array}$ & \\
\hline $\operatorname{TGARCH}(1,1)$ & $\begin{array}{c}0.0000012^{*} \\
(0.0021)\end{array}$ & $\begin{array}{c}0.021040^{*} \\
(0.0000)\end{array}$ & & & & $\begin{array}{c}-0.104025^{\star} \\
(0.0000)\end{array}$ & $\begin{array}{c}1.021107^{*} \\
(0.0000)\end{array}$ & & & \\
\hline
\end{tabular}

Note: $*$ significant at $1 \% ; * *=$ significant at $5 \% ; * * *=$ significant at $10 \%$; value in the parenthesis is the $p$-value 
Table 9. The Results of GARCH Construction Process for White Pepper

\begin{tabular}{|c|c|c|c|c|c|c|c|c|c|c|}
\hline $\begin{array}{l}\text { GARCH-type } \\
\text { Models }\end{array}$ & $\alpha 0$ or $\omega$ & $\alpha 1$ & $\alpha 2$ & $\alpha 3$ & $\alpha 4$ & $\gamma$ & $\beta 1$ & $\beta 2$ & $\beta 3$ & $\beta 4$ \\
\hline $\mathrm{ARCH}$ (1) & $\begin{array}{c}0.000812^{*} \\
(0.0003)\end{array}$ & $\begin{array}{c}0.380639^{\star \star} \\
(0.0421)\end{array}$ & & & & & & & & \\
\hline $\mathrm{ARCH}(2)$ & $\begin{array}{c}0.000433^{*} \\
(0.0000)\end{array}$ & $\begin{array}{c}0.315429^{* *} \\
(0.0289)\end{array}$ & $\begin{array}{c}0.898046^{* *} \\
(0.0333)\end{array}$ & & & & & & & \\
\hline $\operatorname{GARCH}(1,1)$ & $\begin{array}{c}0.000114^{* \star} \\
(0.0300)\end{array}$ & $\begin{array}{c}0.457453^{*} \\
(0.0003)\end{array}$ & & & & & $\begin{array}{c}0.596389^{*} \\
(0.0000)\end{array}$ & & & \\
\hline $\operatorname{GARCH}(2,1)$ & $\begin{array}{c}0.0000555^{\star} \\
(0.0060)\end{array}$ & $\begin{array}{c}0.181129^{*} \\
(0.0004)\end{array}$ & & & & & $\begin{array}{c}1.213938^{*} \\
(0.0000)\end{array}$ & $\begin{array}{c}-0.404459^{\star} \\
(0.0000) \\
\end{array}$ & & \\
\hline $\operatorname{GARCH}(3,1)$ & $\begin{array}{c}0.0000994^{*} \\
(0.0000)\end{array}$ & $\begin{array}{c}0.287268^{*} \\
(0.0010)\end{array}$ & & & & & $\begin{array}{c}0.521475^{*} \\
(0.0000)\end{array}$ & $\begin{array}{c}0.463383^{*} \\
(0.0000)\end{array}$ & \begin{tabular}{|c|}
$-0.291665^{*}$ \\
$(0.0000)$
\end{tabular} & \\
\hline $\operatorname{GARCH}(3,2)$ & $\begin{array}{c}0.000184^{*} \\
(0.0020)\end{array}$ & $\begin{array}{c}0.227343^{\star \star *} \\
(0.0260) \\
\end{array}$ & $\begin{array}{c}0.277182^{\star \star \star} \\
(0.0525) \\
\end{array}$ & & & & $\begin{array}{c}0.596875^{\star} \\
(0.0000)\end{array}$ & $\begin{array}{c}-0.434155^{\star} \\
(0.0002)\end{array}$ & $\begin{array}{c}0.299880^{*} \\
(0.0041)\end{array}$ & \\
\hline GARCH-M $(1,1)$ & $\begin{array}{c}0.000136^{*} \\
(0.0054)\end{array}$ & $\begin{array}{c}0.325286^{*} \\
(0.0006)\end{array}$ & & & & & $\begin{array}{c}0.624171^{*} \\
(0.0000)\end{array}$ & & & \\
\hline GARCH-M $(2,1)$ & $\begin{array}{c}0.000050^{*} \\
(0.0000)\end{array}$ & $\begin{array}{c}0.203357^{*} \\
(0.0000)\end{array}$ & & & & & $\begin{array}{c}1.218667^{*} \\
(0.0000)\end{array}$ & $\begin{array}{c}-0.407980^{\star} \\
(0.0000)\end{array}$ & & \\
\hline GARCH-M $(3,2)$ & $\begin{array}{c}0.000207^{*} \\
(0.0000)\end{array}$ & $\begin{array}{c}0.198722^{*} \\
(0.0083)\end{array}$ & $\begin{array}{c}0.290203^{*} \\
(0.0083)\end{array}$ & & & & $\begin{array}{c}0.409599^{*} \\
(0.0000)\end{array}$ & $\begin{array}{c}-0.336333^{*} \\
(0.0000)\end{array}$ & $\begin{array}{c}0.352438^{*} \\
(0.0000)\end{array}$ & \\
\hline $\operatorname{EGARCH}(3,2)$ & $\begin{array}{c}-3.540747^{\star} \\
(0.0000)\end{array}$ & $\begin{array}{c}0.400504^{*} \\
(0.0032)\end{array}$ & $\begin{array}{c}0.342236^{*} \\
(0.0000)\end{array}$ & & & $\begin{array}{c}-0.110303^{*} \\
(0.0015)\end{array}$ & $\begin{array}{c}0.615059^{*} \\
(0.0000)\end{array}$ & $\begin{array}{c}-0.749592^{*} \\
(0.0000) \\
\end{array}$ & $\begin{array}{c}0.715046^{*} \\
(0.0000) \\
\end{array}$ & \\
\hline $\operatorname{TGARCH}(3,2)$ & $\begin{array}{c}0.000140^{*} \\
(0.0000)\end{array}$ & $\begin{array}{c}0.447094^{*} \\
(0.0003)\end{array}$ & $\begin{array}{c}0.436279^{*} \\
(0.0000)\end{array}$ & & & $\begin{array}{c}-0.271365^{\star *} \\
(0.0412)\end{array}$ & $\begin{array}{c}0.534865^{*} \\
(0.0000)\end{array}$ & $\begin{array}{c}-0.344292^{*} \\
(0.0000)\end{array}$ & $\begin{array}{c}0.224184^{*} \\
(0.0000)\end{array}$ & \\
\hline
\end{tabular}

Note: ${ }^{*}=$ significant at $1 \% ;{ }^{* *}=$ significant at $5 \% ;{ }^{* * *}=$ significant at $10 \%$; value in the parenthesis is the $\mathrm{p}$-value

Table 10. The Results of GARCH Construction Process for Black Pepper

\begin{tabular}{|c|c|c|c|c|c|c|c|c|c|c|}
\hline $\begin{array}{l}\text { GARCH-type } \\
\text { Models }\end{array}$ & $\alpha 0$ or $\omega$ & $\alpha 1$ & $\alpha 2$ & a3 & $\alpha 4$ & $\gamma$ & $\beta 1$ & $\beta 2$ & $\beta 3$ & $\beta 4$ \\
\hline $\mathrm{ARCH}(1)$ & $\begin{array}{c}0.000934^{*} \\
(0.0000)\end{array}$ & $\begin{array}{c}0.11896^{\star \star \star} \\
(0.0763)\end{array}$ & & & & & & & & \\
\hline $\operatorname{GARCH}(1,1)$ & $\begin{array}{c}0.000029^{*} \\
(0.0002)\end{array}$ & $\begin{array}{c}0.061459^{*} \\
(0.0000)\end{array}$ & & & & & $\begin{array}{c}0.918647^{*} \\
(0.0000)\end{array}$ & & & \\
\hline $\operatorname{GARCH}(3,1)$ & $\begin{array}{c}0.0000469^{*} \\
(0.0000)\end{array}$ & $\begin{array}{c}0.048523^{*} \\
(0.0000)\end{array}$ & & & & & $\begin{array}{c}-0.550656^{*} \\
(0.0000)\end{array}$ & $\begin{array}{c}0.510622^{*} \\
(0.0000)\end{array}$ & \begin{tabular}{|c|}
$0.956864^{*}$ \\
$(0.0000)$
\end{tabular} & \\
\hline $\operatorname{GARCH}(1,3)$ & $\begin{array}{c}0.0000901^{*} \\
(0.0019)\end{array}$ & $\begin{array}{c}0.187011^{\text {** }} \\
(0.0307)\end{array}$ & \begin{tabular}{|c|}
$-0.146054^{\star \star \star *}$ \\
$(0.0557)$
\end{tabular} & $\begin{array}{c}0.108639^{*} \\
(0.0004)\end{array}$ & & & $\begin{array}{c}0.786802^{*} \\
(0.0000)\end{array}$ & & & \\
\hline $\operatorname{GARCH}(2,4)$ & $\begin{array}{c}0.00000213^{\star} \\
(0.0017)\end{array}$ & $\begin{array}{c}0.352383^{\star} \\
(0.0008)\end{array}$ & $\begin{array}{c}-0.658199^{\star} \\
(0.0011)\end{array}$ & $\begin{array}{c}0.369480^{\star} \\
(0.0012)\end{array}$ & $\begin{array}{c}-0.070149^{\star \star \star \star} \\
(0.0823) \\
\end{array}$ & & $\begin{array}{c}1.842263^{\star} \\
(0.0000)\end{array}$ & \begin{tabular}{|c|}
$-0.838006^{\star}$ \\
$(0.0000)$ \\
\end{tabular} & & \\
\hline GARCH-M $(1,1)$ & $\begin{array}{c}0.0000277^{*} \\
(0.0003)\end{array}$ & $\begin{array}{c}0.062022^{\star} \\
(0.0000)\end{array}$ & & & & & $\begin{array}{c}0.919109^{*} \\
(0.0000)\end{array}$ & & & \\
\hline GARCH-M $(3,1)$ & $\begin{array}{c}0.0000615^{\star} \\
(0.0000)\end{array}$ & $\begin{array}{c}0.048801^{\star} \\
(0.0000)\end{array}$ & & & & & $\begin{array}{c}-0.557928^{\star} \\
(0.0000)\end{array}$ & $\begin{array}{c}0.515412^{*} \\
(0.0000)\end{array}$ & \begin{tabular}{|c|}
$0.932797^{\star}$ \\
$(0.0000)$ \\
\end{tabular} & \\
\hline GARCH-M $(4,1)$ & $\begin{array}{c}0.0000585^{\star} \\
(0.0001)\end{array}$ & $\begin{array}{c}0.078052^{\star} \\
(0.0000)\end{array}$ & & & & & $\begin{array}{c}0.717098^{*} \\
(0.0000)\end{array}$ & \begin{tabular}{|c|}
$-0.284087^{*}$ \\
$(0.0001)$
\end{tabular} & \begin{tabular}{|c|}
$-0.276544^{*}$ \\
$(0.0003)$
\end{tabular} & $\begin{array}{c}0.702511^{*} \\
(0.0000)\end{array}$ \\
\hline GARCH-M $(2,2)$ & $\begin{array}{c}0.0000266^{*} \\
(0.0017)\end{array}$ & $\begin{array}{c}0.349700^{*} \\
(0.0009)\end{array}$ & $\begin{array}{c}-0.26324^{*} \\
(0.0096) \\
\end{array}$ & & & & $\begin{array}{c}0.680103^{*} \\
(0.0000)\end{array}$ & $\begin{array}{c}0.226444^{*} \\
(0.0080)\end{array}$ & & \\
\hline GARCH-M $(2,4)$ & $\begin{array}{c}0.00000331^{*} \\
(0.0000)\end{array}$ & $\begin{array}{c}0.152169^{*} \\
(0.0000)\end{array}$ & $\begin{array}{c}-0.249642^{\star} \\
(0.0000)\end{array}$ & $\begin{array}{c}0.237501^{*} \\
(0.0000)\end{array}$ & $\begin{array}{c}-0.141869^{*} \\
(0.0000)\end{array}$ & & $\begin{array}{c}1.682531^{*} \\
(0.0000)\end{array}$ & \begin{tabular}{|c|}
$-0.683137^{*}$ \\
$(0.0000)$
\end{tabular} & & \\
\hline $\operatorname{EGARCH}(1,1)$ & $\begin{array}{c}-0.205857^{*} \\
(0.0000)\end{array}$ & $\begin{array}{c}-0.133261^{*} \\
(0.0000)\end{array}$ & & & & $\begin{array}{c}0.014694^{* *} \\
(0.0249)\end{array}$ & $\begin{array}{c}0.956663^{*} \\
(0.0000)\end{array}$ & & & \\
\hline $\operatorname{EGARCH}(3,1)$ & $\begin{array}{c}-1.312015^{*} \\
(0.0000)\end{array}$ & $\begin{array}{c}0.204348^{*} \\
(0.0000)\end{array}$ & & & & $\begin{array}{c}0.133665^{\star} \\
(0.0000)\end{array}$ & $\begin{array}{c}-0.573671^{*} \\
(0.0000)\end{array}$ & $\begin{array}{c}0.460569^{*} \\
(0.0000)\end{array}$ & \begin{tabular}{|c|}
$0.943783^{\star}$ \\
$(0.0000)$ \\
\end{tabular} & \\
\hline $\operatorname{EGARCH}(4,1)$ & $\begin{array}{c}-1.054662^{\star} \\
(0.0000)\end{array}$ & $\begin{array}{c}0.276511^{*} \\
(0.0000)\end{array}$ & & & & $\begin{array}{c}0.201118^{\star} \\
(0.0000)\end{array}$ & $\begin{array}{c}-0.162038^{\star \star \star *} \\
(0.0536)\end{array}$ & \begin{tabular}{|c|}
$0.686271^{*}$ \\
$(0.0000)$
\end{tabular} & \begin{tabular}{|c|}
$0.745626^{\star}$ \\
$(0.0000)$ \\
\end{tabular} & $\begin{array}{c}-0.392487^{\star} \\
(0.0000)\end{array}$ \\
\hline EGARCH $(2,3)$ & $\begin{array}{c}-0.159932^{\star} \\
(0.0000)\end{array}$ & $\begin{array}{c}0.176954^{\star} \\
(0.0000)\end{array}$ & $\begin{array}{c}-0.341919^{*} \\
(0.0000)\end{array}$ & $\begin{array}{c}0.020034^{\star} \\
(0.0047)\end{array}$ & & $\begin{array}{c}0.013143^{\star} \\
(0.0001)\end{array}$ & $\begin{array}{c}1.031187^{\star} \\
(0.0000)\end{array}$ & \begin{tabular}{|c|}
$-0.068945^{*}$ \\
$(0.0000)$
\end{tabular} & & \\
\hline $\operatorname{TGARCH}(3,1)$ & $\begin{array}{c}0.000123^{*} \\
(0.0013)\end{array}$ & $\begin{array}{c}0.159020^{*} \\
(0.0000)\end{array}$ & & & & $\begin{array}{c}0.192874^{* * *} \\
(0.0602)\end{array}$ & $\begin{array}{c}-0.163370^{*} \\
(0.0000)\end{array}$ & $\begin{array}{c}0.343563^{*} \\
(0.0000)\end{array}$ & \begin{tabular}{|c|}
$0.505668^{*}$ \\
$(0.0000)$
\end{tabular} & \\
\hline TGARCH $(1,2)$ & $\begin{array}{c}0.000176^{*} \\
(0.0000)\end{array}$ & $\begin{array}{c}0.101658^{\star \star \star} \\
(0.0868)\end{array}$ & $\begin{array}{c}0.123224^{*} \\
(0.0054)\end{array}$ & & & $\begin{array}{c}-0.199964^{*} \\
(0.0001)\end{array}$ & $\begin{array}{c}0.686407^{\star} \\
(0.0000)\end{array}$ & & & \\
\hline TGARCH $(3,2)$ & $\begin{array}{c}0.0000033^{*} \\
(0.0000)\end{array}$ & $\begin{array}{c}0.173174^{*} \\
(0.0000)\end{array}$ & $\begin{array}{c}-0.178141^{\text {** }} \\
(0.0000)\end{array}$ & & & $\begin{array}{c}-0.008977^{\star \star} \\
(0.0109)\end{array}$ & $\begin{array}{c}1.364921^{*} \\
(0.0000)\end{array}$ & $\begin{array}{c}-0.067466^{\star *} \\
(0.0122)\end{array}$ & $\begin{array}{c}-0.291808^{*} \\
(0.0000)\end{array}$ & \\
\hline
\end{tabular}

Note: $*=$ significant at $1 \% ; * *=$ significant at $5 \% ; * * *=$ significant at $10 \%$; value in the parenthesis is the p-value 
Table 11. The Summary of Best GARCH-Type Models

\begin{tabular}{|c|c|c|c|c|c|c|c|c|c|c|c|c|}
\hline Commodities & $\begin{array}{l}\text { Evaluation } \\
\text { Statistics }\end{array}$ & GARCH-type Models & $\alpha 0$ or $\omega$ & a1 & a2 & a3 & a4 & $\nu$ & $\beta 1$ & $\beta 2$ & $\beta 3$ & $\beta 4$ \\
\hline \multirow{2}{*}{$\mathrm{CPO}$} & $\begin{array}{l}\text { RMSE } \\
\text { MAE }\end{array}$ & GARCH-M $(3,3)$ & $\begin{array}{c}0.000158^{\text {t* }} \\
(0.0412)\end{array}$ & $\begin{array}{c}0.134362^{* *} \\
(0.0215) \\
\end{array}$ & $\begin{array}{c}-0.097256^{* *} \\
(0.0309)\end{array}$ & $\begin{array}{c}0.175471^{*} \\
(0.0021) \\
\end{array}$ & & & $\begin{array}{c}0.695960^{*} \\
(0.0000) \\
\end{array}$ & $\begin{array}{c}-0.635278^{*} \\
(0.0000) \\
\end{array}$ & $\begin{array}{c}0.622947^{*} \\
(0.0000)\end{array}$ & \\
\hline & MAPE & $\operatorname{TGARCH}(4,2)$ & $\begin{array}{c}0.000169^{*} \\
(0.0000)\end{array}$ & $\begin{array}{c}0.182262^{*} \\
(0.0001)\end{array}$ & $\begin{array}{c}0.077179^{*+*} \\
(0.0565)\end{array}$ & & & $\begin{array}{c}-0.058606^{\text {ta**}} \\
(0.0582)\end{array}$ & $\begin{array}{c}-0.303633^{\text {t* }} \\
(0.0261)\end{array}$ & $\begin{array}{c}0.951457^{*} \\
(0.0000)\end{array}$ & $\begin{array}{c}0.507213^{*} \\
(0.0000)\end{array}$ & $\begin{array}{c}-0.484314^{*} \\
(0.0002)\end{array}$ \\
\hline \multirow{2}{*}{$\begin{array}{l}\text { Natural Rubber } \\
\text { TSR20 }\end{array}$} & $\begin{array}{l}\text { RMSE } \\
\text { MAE }\end{array}$ & $\operatorname{EGARCH}(3,1)$ & $\begin{array}{c}-1.139105^{*} \\
(0.0032)\end{array}$ & $\begin{array}{c}0.261171^{*} \\
(0.0000)\end{array}$ & & & & $\begin{array}{c}-0.120674^{*} \\
(0.0052)\end{array}$ & $\begin{array}{c}1.623358^{*} \\
(0.0000)\end{array}$ & $\begin{array}{c}-1.601765^{*} \\
(0.0000) \\
\end{array}$ & $\begin{array}{c}0.822213^{*} \\
(0.0000)\end{array}$ & \\
\hline & MAPE & $\operatorname{EGARCH}(1,1)$ & $\begin{array}{c}-2.055568^{*} \\
(0.0047)\end{array}$ & $\begin{array}{c}0.416561^{*} \\
(0.0012)\end{array}$ & & & & $\begin{array}{c}-0.186586^{\text {ts** }} \\
(0.0853)\end{array}$ & $\begin{array}{c}0.710663^{*} \\
(0.0000)\end{array}$ & & & \\
\hline \multirow{2}{*}{ Arabica Coffee } & $\begin{array}{l}\text { RMSE } \\
\text { MAE }\end{array}$ & $\operatorname{EGARCH}(4,1)$ & $\begin{array}{c}-16.677680^{*} \\
(0.0000)\end{array}$ & $\begin{array}{c}0.036383^{* \pm *} \\
(0.0610)\end{array}$ & & & & $\begin{array}{c}0.067865^{*} \\
(0.0001)\end{array}$ & $\begin{array}{c}0.578589^{*} \\
(0.0000)\end{array}$ & $\begin{array}{c}-1.790400^{*} \\
(0.0000)\end{array}$ & $\begin{array}{c}0.544335^{*} \\
(0.0000)\end{array}$ & $\begin{array}{c}-0.794044^{*} \\
(0.0000)\end{array}$ \\
\hline & MAPE & GARCH-M $(2,1)$ & $\begin{array}{c}0.001144^{*} \\
(0.0000)\end{array}$ & $\begin{array}{c}-0.021503^{\text {t* }} \\
(0.0443)\end{array}$ & & & & & $\begin{array}{c}1.149463^{*} \\
(0.0000)\end{array}$ & $\begin{array}{c}-0.992513^{*} \\
(0.0000)\end{array}$ & & \\
\hline \multirow{2}{*}{ Robusta Coffee } & $\begin{array}{l}\text { RMSE } \\
\text { MAE }\end{array}$ & $\operatorname{EGARCH}(1,3)$ & $\begin{array}{c}-0.029515^{*} \\
(0.0000)\end{array}$ & $\begin{array}{c}-0.039061^{*} \\
(0.0000)\end{array}$ & $\begin{array}{c}0.231862^{*} \\
(0.0000)\end{array}$ & $\begin{array}{c}-0.263604^{*} \\
(0.0000)\end{array}$ & & $\begin{array}{c}0.038077^{\text {t*** }} \\
(0.0860)\end{array}$ & $\begin{array}{c}0.987449^{*} \\
(0.0000)\end{array}$ & & & \\
\hline & MAPE & $\operatorname{GARCH}(3,2)$ & $\begin{array}{c}0.000686^{* t} \\
(0.0140)\end{array}$ & $\begin{array}{c}0.050870^{*} \\
(0.0017)\end{array}$ & $\begin{array}{c}0.108399^{*} \\
(0.0000)\end{array}$ & & & & $\begin{array}{c}-0.13602^{\star} \\
(0.0000)\end{array}$ & $\begin{array}{c}-0.091606^{\text {t* }} \\
(0.0281)\end{array}$ & $\begin{array}{c}0.821833^{*} \\
(0.0000)\end{array}$ & \\
\hline \multirow{2}{*}{ Cocoa } & $\begin{array}{l}\text { RMSE } \\
\text { MAE }\end{array}$ & $\operatorname{EGARCH}(3,2)$ & $\begin{array}{c}-1.398572^{+*+*} \\
(0.0619)\end{array}$ & $\begin{array}{c}0.284362^{*} \\
(0.0000)\end{array}$ & $\begin{array}{c}0.268115^{*} \\
(0.0000)\end{array}$ & & & $\begin{array}{c}0.064278^{*} \\
(0.0006)\end{array}$ & $\begin{array}{c}-0.808895^{*} \\
(0.0000) \\
\end{array}$ & $\begin{array}{c}0.718652^{*} \\
(0.0000) \\
\end{array}$ & $\begin{array}{c}0.933300^{*} \\
(0.0000) \\
\end{array}$ & \\
\hline & MAPE & GARCH-M $(3,2)$ & $\begin{array}{c}0.00000127^{*} \\
(0.0000)\end{array}$ & $\begin{array}{c}0.099086^{*} \\
(0.0097)\end{array}$ & $\begin{array}{c}-0.099106^{* *} \\
(0.0111)\end{array}$ & & & & $\begin{array}{c}0.973997^{*} \\
(0.0000)\end{array}$ & $\begin{array}{c}0.908512^{*} \\
(0.0000)\end{array}$ & $\begin{array}{c}-0.883058^{*} \\
(0.0000)\end{array}$ & \\
\hline \multirow{3}{*}{ White Pepper } & RMSE & ARCH 1 & $\begin{array}{c}0.000812^{*} \\
(0.0003)\end{array}$ & $\begin{array}{c}0.380639^{* *} \\
(0.0421)\end{array}$ & & & & & & & & \\
\hline & MAPE & $\operatorname{EGARCH}(3,2)$ & $\begin{array}{c}-3.540747^{*} \\
(0.0000)\end{array}$ & $\begin{array}{c}0.400504^{*} \\
(0.0032)\end{array}$ & $\begin{array}{c}0.342236^{*} \\
(0.0000)\end{array}$ & & & $\begin{array}{c}-0.110303^{*} \\
(0.0015) \\
\end{array}$ & $\begin{array}{c}0.615059^{*} \\
(0.0000)\end{array}$ & $\begin{array}{c}-0.749592^{*} \\
(0.0000) \\
\end{array}$ & $\begin{array}{c}0.715046^{*} \\
(0.0000) \\
\end{array}$ & \\
\hline & MAE & GARCH-M $(1,1)$ & $\begin{array}{c}0.000136^{*} \\
(0.0054)\end{array}$ & $\begin{array}{c}0.325286^{*} \\
(0.0006)\end{array}$ & & & & & $\begin{array}{c}0.624171^{*} \\
(0.0000)\end{array}$ & & & \\
\hline \multirow{2}{*}{ Black Pepper } & RMSE & $\operatorname{EGARCH}(3,4)$ & $\begin{array}{c}-0.627067^{*} \\
(0.0000)\end{array}$ & $\begin{array}{c}0.372568^{*} \\
(0.0015)\end{array}$ & $\begin{array}{c}-0.170544^{*} \\
(0.0000)\end{array}$ & $\begin{array}{c}-0.280792^{*} \\
(0.0000)\end{array}$ & $\begin{array}{c}-0.418333^{*} \\
(0.0011)\end{array}$ & $\begin{array}{c}-0.069481^{\text {t* }} \\
(0.0196)\end{array}$ & $\begin{array}{c}-0.209616^{*} \\
(0.0000)\end{array}$ & $\begin{array}{l}0.14391^{*} \\
(0.0000)\end{array}$ & $\begin{array}{c}0.926304^{*} \\
(0.0000)\end{array}$ & \\
\hline & $\begin{array}{l}\text { MAPE } \\
\text { MAE }\end{array}$ & $\operatorname{EGARCH}(2,3)$ & $\begin{array}{c}-0.159932^{*} \\
(0.0000)\end{array}$ & $\begin{array}{c}0.176954^{*} \\
(0.0000)\end{array}$ & $\begin{array}{c}-0.341919^{*} \\
(0.0000)\end{array}$ & $\begin{array}{c}0.020034^{*} \\
(0.0047)\end{array}$ & & $\begin{array}{c}0.013143^{*} \\
(0.0001) \\
\end{array}$ & $\begin{array}{c}1.031187^{*} \\
(0.0000)\end{array}$ & $\begin{array}{c}-0.068945^{*} \\
(0.0000)\end{array}$ & & \\
\hline
\end{tabular}

be used to predict the volatility of spot price returns of CPO. Table 5 shows that ARCH model; GARCH model; GARCH-M model; and EGARCH model can be used to predict the volatility of spot price returns of TSR20. Table 6 shows that GARCH model; GARCH-M model; EGARCH model; and TGARCH model can be used to predict the volatility of spot price returns of Arabica Coffee. Table 7 shows that GARCH model; GARCH-M model; EGARCH model; and TGARCH model can be used to predict the volatility of spot price returns of Robusta Coffee. Similar to Arabica Coffee and Robusta Coffee, ARCH model also cannot be used to predict the volatility of Cocoa spot price returns. Table 8 shows that GARCH model; GARCH-M model; EGARCH model; and TGARCH model can be used to predict the volatility of spot price returns of Cocoa. Table 9 shows that ARCH model; GARCH model; GARCH-M model; EGARCH model; and TGARCH model, can be used to predict the volatility of spot price returns of White Pepper. Similar to White Pepper, all of the GARCH-type models in this study were fit as volatility prediction models for Black Pepper's spot price returns volatility.

This study has chosen the most recent 67 weeks, which are about $20 \%$ of total observations of each commodity, as the periods to evaluate the predictability of the significant GARCH-type models (Brook, 2008). The evaluation process results are shown in Table 11.

With respect to RMSE criterion, $\mathrm{ARCH}$ is the best prediction model for returns volatility of White
Pepper; GARCH-M is the best prediction model for returns volatility of $\mathrm{CPO}$; and EGARCH is the best prediction model for returns volatility of Natural Rubber TSR20, Arabica Coffee, Robusta Coffee, Cocoa and Black Pepper. In this criterion, GARCH model and TGARCH model are not selected as the best prediction model of any commodities.

With respect to MAPE criterion, GARCH is the best prediction model for returns volatility of Robusta Coffee; GARCH-M is the best prediction model for returns volatility of Arabica Coffee and Cocoa; EGARCH is the best prediction model for returns volatility of TSR20, White Pepper and Black Pepper; TGARCH was the best prediction model for returns volatility of CPO. In this criterion, $\mathrm{ARCH}$ is not selected as the best prediction model of the returns volatility of any commodities.

With respect to MAE criterion, GARCH-M is the best prediction model for returns volatility of $\mathrm{CPO}$ and White Pepper; and EGARCH is the best prediction model for returns volatility of TSR20, Arabica Coffee, Robusta Coffee, Cocoa and Black Pepper. In this criterion, ARCH model, GARCH model and TGARCH model are not selected as the best prediction models of the returns volatility of any commodities.

The predictability of ARCH model in Indonesian exported agricultural commodities, as had been discussed in this study, is supported by the studies of Beck (2001) and Sumaryanto (2009). Their studies found that ARCH model was fit to predict the volatility of commodity price returns. 
Furthermore, the predictability of GARCH model in Indonesian exported agricultural commodities is supported by Beck (2001), Yang et al. (2001), Swaray (2002), Zheng et al. (2008), Sumaryanto (2009), Pinisakikool (2009), O'Connor et al. (2009) and Mahesha (2011). EGARCH model is found as the best model in predicting the spot price returns volatility of Natural Rubber TSR20, Arabica Coffee, Robusta Coffee, Cocoa, White Pepper and Black Pepper. It seemed that EGARCH model is the best prediction model for all commodities, except CPO. The predictability of EGARCH Model in predicting the returns volatility of Indonesia exported agricultural commodities is supported by the studies of Swaray (2002) and Zheng et al. (2008). TGARCH model is found as the best model in predicting the returns volatility of CPO. The result is supported by studies of Huang et al. (2008) and Pinisakikool (2009).

\section{CONCLUSIONS}

High level of volatility can indicate that a commodity has a high risk. The results of this study can give benefit to investor and prospective investors to manage their portfolios and asses their investment risks related to those seven commodities. In order to deal with the relatively high volatility level of Indonesia commodities spot price returns, financial instruments such as forwards and futures markets may be desirable. The results in this study give insight to the market players about timing of hedging.

The information in this study also can give additional information to Indonesian government in increasing cash inflow to the country, since Indonesia is an international major player in those seven commodities markets (Agriculture Data Center, 2011). The increase of cash inflow can be realized by maintaining the existence of the commodities, quantities and quality, in fulfill the market demand. The welfare of farmers and small private sectors of those seven commodities should be put into account. Government can give them insight and encouragement to add values of those commodities through manufacturing sector. They can manufacture those commodities further to be another half or full finished goods forms. Therefore, when the market prices become too high which lead to the decrease of demand, they will survive from the manufactured products of those commodities.

The information from this study can also be a useful reference for economist, financial analysts and researchers, who are interested in Indonesian agricultural export commodities and also interested in the application of GARCH-type models.
The application of GARCH-type models in agricultural fields could be a significant contribution to quantitative analysis of financial fields.

For the future researches, the prediction of risk by GARCH-type models used in this study could also be applied in other research objects, such as fixed income financial asset markets, currency markets, stock markets, other commodities markets, tourism, etc. The future researches also can use advanced type of GARCH models in order to get more specific results.

\section{REFERENCES}

Agriculture Data Center (2011), Monthly Bulletin: Indikator Makro Sektor Pertanian, (Macro Indicators of Agricultural Sector), Jakarta: Data Center and Information System of Indonesian Agriculture Ministry, August Edition.

Alom, M.D.F., Ward, B.D. \& Hu, Baiding (2010), "Cross Country Mean and Volatility Spillover Effects of Food Prices: Evidence for Asia and Pacific", International Review of Business Research Papers, 6 (2), 334-355.

Apergis, N., \&Rezitis, A. (2003), "Agricultural Price Volatility Spillover Effects: The Case of Greece". Journal of Agricultural and Applied Economics, 43, 95-110.

Apergis, N., \&Rezitis, A. (2011), "Food Price Volatility and Macroeconomic Factors: Evidence from GARCH and GARCH-X Estimates", European Review of Agricultural Economics, 30, 389-406.

Basri, F. (2002), Perekonomian Indonesia (Indonesian Economy), Jakarta: Erlangga.

Beck, Stacie (2001), "Autoregressive Conditional Heterocedasticity in Commodity Spot Prices", Journal of Applied Econometrics, 16, 115-132.

Bollerslev, T. (1986), "Generalized Autoregressive Conditional Heteroscedasticity", Journal of Econometrics, 31, 307-327.

Brooks, Chris. (2008), Introductory Econometrics for Finance, New York: Cambridge University Press, $2^{\text {nd }}$ edition.

Commodity Futures Trading Regulatory Agency (CoFTRA) (2011), Forward-Futures-Spot Commodities Values Data, Retrieved July 04, 2011 from http://www.bappebti.go.id/pg=harga_bursa.

Daryanto, Arief (1999), Indonesia's Crisis and the Agricultural Sector: the Relevance of Agricultural Demand-Led Industrialization, UNEAC Asia Paper, 2. Australia.

Deaton, Angus (1999), "Commodity Prices and Growth in Africa", Journal of Economic Perspectives, 13, 23-40.

Diebold, F.X. (2004), "The Nobel Prize for Robert F. Engle", Scandinavian Journal of Economics, 106, 165-185. 
Djamin, Zulkarnain (1989), Perekonomian Indonesia (Indonesian Economy), Jakarta: Published Center of Economics Faculty, University of Indonesia.

Engle, R.F. (1982), "Autoregressive Conditional Heteroscedasticity with Estimates of the Variance of United Kingdom Inflation", Econometrica, 50, 987-1007.

Engle, R.F. (2003), Risk and Volatility: Econometric Models and Financial Practice, Nobel Prize in Economics Documents 2003-4, Nobel Prize Committee.

Engle, R.F., Lilien, L.D., \& Robins, R. (1987), "Estimation of Time Variying Risk Premiums in the Term Structure", Econometrica, 55, 391-408.

Francq, C., Zakoian, J.M. (2010), Garch Models: Structure, Statistical Inference and Financial Applications, Wiley.

Huang, B.W., Yeh, C.Y., Chen, M.G., Lin, Y.Y. \& Shih, M.L. (2008), Threshold and Asymmetric Volatility in Taiwan Broiler Farm Price Change, In Proceedings of the Third International Conference on Convergence and Hybrid Information Technology (pp. 10371042), Retrieved from IEEE Computer Society (DOI 10.1109/ICCIT.2008.71).

Kroner, Kenneth F., Kneafsey, Kevin P. \& Claessens, S. (1995), "Forecasting Volatility in Commodity Markets", Journal of Forecasting, 14, 77-95.

Mahesha, M. (2011), "International Price Volatility of Indian Spices Exports-An Empirical Analysis Asia and Pacific", Journal of Research in Business Management, 2, 110-116.

Nelson, D.B. (1991), "Conditional Heteroskedasticity in Asset Returns: A New Approach", Econometrica, 59(2), 347-370.

Newbery, D.M. (1989), "The Theory of Food Price Stabilization", The Economic Journal, 9, 1065-1082.

O’Connor, D., Keane, M. \& Barner, E. (2009), Measuring Volatility in Dairy Commodity Prices. The $113^{\text {th }}$ European Association of Agricultural Economist Seminar, Greece, 1-16.

Pinisakikool, Teerapong (2009), "Do Futures Stabilize the Volatility of the Agricultural Spot Prices? Evidence from Thailand", Euro Economica, 22 (1), 47-56.
Poon, S.H., and Granger, J. (2003), "Forecasting Volatility in Financial Markets: A Review", Journal of Economic Literature, XIL, 478-539.

Sekhar, C.S.C. (2003), Volatility of Agricultural Prices-An Analysis of Major International and Domestic Markets, Indian Council for Research on International Economic Relations, Working Paper No. 103.

Sekhar, C.S.C. (2004), "Agricultural Price Volatility in International and Indian Markets", Economic and Political Weekly, 39, 4729-4736.

Shephard, N. (1996), Statistical Aspect of ARCH and Stochastic Volatility, In Cox, D.R., Hinkley, E.V., and Barndoff-Nielson, O.E. (Eds), Time Series Models in Econometrics, Finance and Other Fields, New York: Chapman and Hall.

Sumaryanto (2009), "Retail Price Volatility Analysis of Some Food Commodities Using ARCH/ GARCH Model", Journal of Agro Economic Indonesia, 27, 125-163.

Swaray, R.B. (2002), Volatility of Primary Commodity Prices: Some Evidence from Agricultural Exports in Sub-Saharan Africa, The University of York, Discussion Papers in Economics, 06.

Taylor, S.J. (1986), Modelling Financial Rime Series. Chichester: John Wiley.

The Agriculture and Natural Resources Team of the UK Department for International Development (DFID) (2004), Rethinking Tropical Agricultural Commodities, The United Kingdom: The Report of DFID.

The World Bank (2011), Agriculture, value added (\% of GDP), World Bank national accounts data, and OECD National Accounts data files, Retrieved November, 02, 2011 from http:// data.worldbank.org/indicator/NV.AGR.TOTL. ZS/countries/ID?display=graph.

Yang, J., Haigh, Michael S. \& Leatham, David J. (2001), "Agricultural Liberalization Policy and Commodity Price Volatility”, Applied Economic Letters, 8, 593-598.

Zakoian, J.M. (1994), "Threshold Heteroscedastic Models", Journal of Economic Dynamic and Control, 12, 193-202.

Zheng, Y., Kinnucan, H.W. \& Thompson, H. (2008), "News and Volatility of Food Prices", Applied Economics, 40, 1629-1635. 